\title{
Profil Kaydırmalı Helisel Dişli Çarkların Matematik Modellenmesi
}

\author{
Cüneyt Fetvaci*
}

\section{ÖZ}

Bu çalışmada evolvent profilli helisel dişli çarkların kremayer takımla imalatının matematik modellenmesi ele alınmıştır. Litvin'in vektör yaklaşımından hareketle takım ve imal edilen dişli çark geometrisini tayin eden ifadeler verilmiştir. Modellemede profil kaydırma ve asimetrik diş profili de göz önüne alınmıştır. İmal edilen dişlide evolvent bölgenin üst sınırının analitik tayini araştırılmıştır. Bir bilgisayar programı geliştirilerek tasarım parametrelerinin imal edilen dişli geometrisindeki etkileri incelenmiştir.

Anahtar Kelimeler: Kremayer takım, asimetrik diş profili, helisel dişli, profil kaydırma

\section{Mathematical Modelling of the Profile Shifted Helical Gears}

\begin{abstract}
This paper studies the mathematical modelling helical gears manufactured by rack cutters. Based on Litvin's vector approach the equations that determine the geometries of cutter and generated gears are given. Addendum modification and asymmetric tooth profile are also taken into consideration in the mathematical model. Analytic determination of involute parameter upper limit is investigated. A computer program is developed to generate the tooth profile of involute helical gears and to illustrate the effect of tool geometry on the generated surfaces.
\end{abstract}

Keywords: Rack cutter, asymmetric tooth profile, helical gear, addendum modification

\footnotetext{
* İletişim Yazarı

Geliş/Received : : 24.06.2019

Kabul/Accepted : :07.10.2019
}

Prof. Dr., İstanbul Üniversitesi, Mühendislik Fakültesi, Makina Mühendisliği Bölümü, İstanbul fetvacic@istanbul.edu.tr, ORCID: 0000-0002-1622-1583 


\section{GÍRİŞ}

Birçok makinanın hayati elemanı olan dişli çarklar otomobillerden uçaklara, ofis makinalarından takım tezgahlarına geniş bir uygulama alanında kullanılmaktadır. Mil eksenlerinin konumuna göre farklı tipleri olmakla birlikte dişli çarkların yaygın uygulama alanı paralel eksenli millerde güç ve hareket iletimidir. Bu uygulamalarda dişler düz veya helisel olarak imal edilmektedir. Düz dişliler tasarım, imalat ve analiz kolaylığı arz ederken, helisel dişlilerdeki tedrici kavrama karakteristiği düz dişlilere nazaran sessiz çalışma imkanı sağlamaktadır. Böylelikle helisel dişliler daha yüksek hızlarda gürültüsüz çalışmaktadır. Helisel dişlilerin diğer bir avantajı alttan kesme olmaksızın daha küçük diş sayılarına inilebilmesidir [1].

Dişli çarkların matematik modellenmesi çok sayıda araştırmaya konu olmuştur. Diş profili evolvent yanak, trokoid kök ve dairesel tabandan oluşmaktadır. Kremayer-tipi ve pinyon-tipi takımlarla imal edilen diş profillerinin matematik modelleri literatürde çeşitli yaklaşımlarla sunulmuştur. Bununla birlikte yaygın olarak kullanılan metod “Litvin'in Vektör Yaklaşımı" dır [2]. Bu metotta ilk olarak takımın imal eden yüzeyleri vektör formda modellenmektedir. Genellikle bu model takımın normal kesitinde tesis edilir. Dişli tipine göre uygun dönüşümler ile farklı kesitlere aktarılabilir. Takım ile taslak arasında kinematik bağ uygun koordinat dönüşümü ile ifade edilir. Diferansiyel geometri ve dişli ana kanunu uygulanarak taslağın dönme parametresi tayin edilir ve sonuç olarak imal edilen dişli geometrisi elde edilir. Ayrıca çeşitli modifikasyonlar (bombeli diş, protuberans vs) modele kolaylıkla eklenebilmektedir. Son yıllarda önem kazanan bir diğer modifikasyon ise dişlerin sağ ve sol profillerinin farklı kavrama açısı ile tasarlanmasıdır. Bu inovatif tasarımla imal edilen dişliler asimetrik dişli çarklar olarak adlandırılır. Hafiflik, yüksek verimlilik, sessizlik ve yüksek güvenilirlik elde edilir [3].

Evolvent profilli dişlilerde küçük diş sayılarında ortaya çıkan alttan kesme diş dibi kesitini zayıflatarak eğilme mukavemetini azaltmakta ve aktif profili kısaltarak kavrama oranını düşürmektedir. Bu nedenle dişlilerin alttan kesilmesi istenmez. Profil kaydırma alttan kesmeyi önlemek için tercih edilir çünkü diğer metotlar özel takım gerektirir.

Literatürde vektör metodunu kullanarak kremayer takımla evolvent düz ve helisel dişli çarkların matematik modellenmesi ve simülasyonunu ele alan birçok çalışma mevcuttur [4-9]. Bu çalışmaların genelinde dikkat çeken bir husus evolvent bölge parametresinin üst sınırının takım geometrisi için geçerli olduğu fakat imal edilen dişlide profilin standart diş başı çapını geçerek sonlanmasıdır. Bu durumda fazla kısmın giderilmesi ya ilave bir algoritma ile ya da CAD programında trim işlemi ile yapılır. Takımla taslağın eş çalışmasında kavrama kıtasında katedilen mesafeden hareketle evolvent parametresinin imal edilen dişlide tam profili sağlayan üst sınırı tayin edilebilir. Fetvacı, gerek kremayer takım ve gerekse pinyon-tipi takım için analitik ifade- 
leri sunmuştur $[10,11]$.

Bu makalede evolvent profilli helisel dişli çarkların kremayer takımla imalatının matematik modellenmesi ele alınmıştır. Takip eden bölümde kesici takımın imal edici yüzeylerinin matematiksel ifadeleri vektör formda verilmiştir. Üçüncü bölümde imal edilen dişli çarkın matematik modeli verilmiştir. Dördüncü bölümde kavrama kıtasında kat edilen mesafeden hareketle evolvent bölgenin üst sınırının analitik tayini açıklanmıştır. Matematik modelden hareketle geliştirilen programın çalıştırmasıyla elde edilen dişli çark grafikleri beşinci bölümde verilmiştir. Sonuçlar altıncı bölümde vurgulanmıştır.

\section{KREMAYER TAKIMIN MATEMATIK MODELI}

\subsection{Normal Kesitte Takım}

Asimetrik evolvent dişli kremayer takım normal kesitte Şekil 1'de gösterilmiştir. Literatürde çeşitli düzenlemelerle verilen ifadeler bu çalışmada sivri uç ve tam yuvarlak uç durumunda da $h_{f}=1.25 \cdot m_{n}$ standart takım baş yüksekliğini sağlayacak şekilde sunulmaktadır $[6,8,12]$. Ayrıca profil kaydırma yer vektörü ifadelerine $e=x \cdot m_{n}$ olarak eklenmiştir. Normal modül $m_{n}$ sembolüyle ve profil kaydırma faktörü $x$ sembolüyle gösterilmektedir.

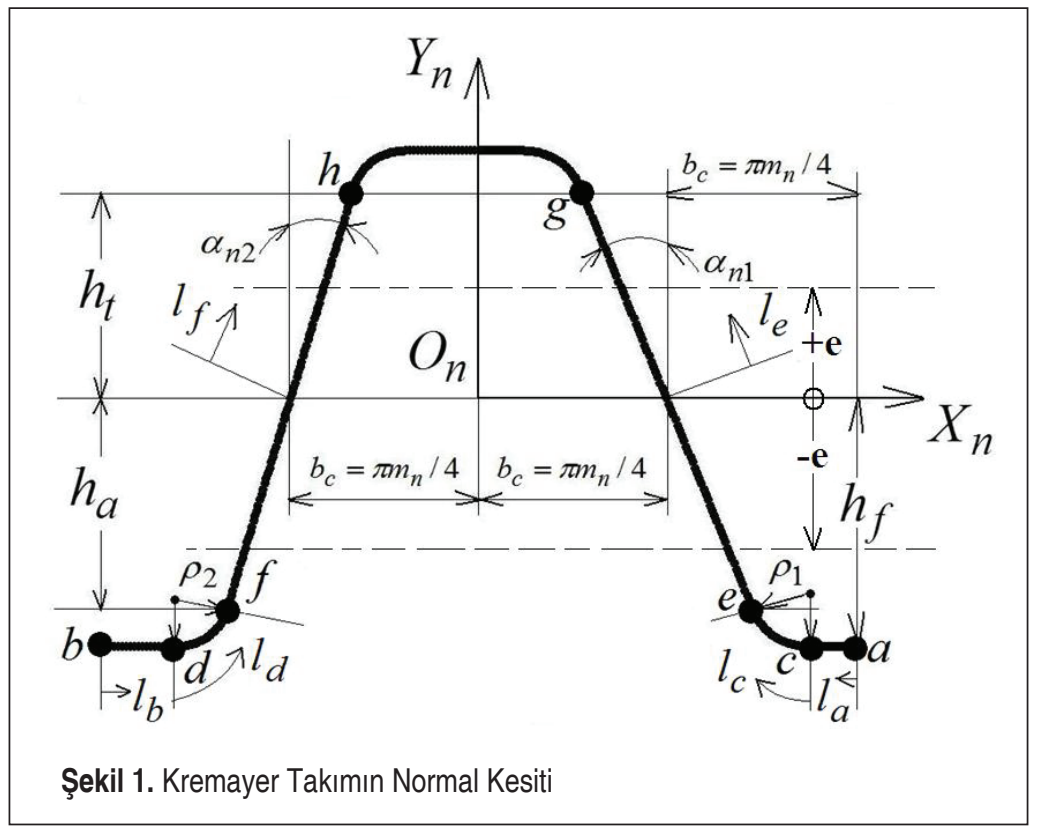


Takımın $\overline{a c}$ ve $\overline{b d}$ bölgeleri imal edilen dişli çarkın taban yüzeylerini sırasıyla oluşturmaktadır. $\quad S_{n}\left(X_{n}, Y_{n}, Z_{n}\right)$ koordinat sisteminde $\overline{a c}$ ve $\overline{b d}$ bölgelerinin yer vektörleri aşağıda verilmiştir. Bölgelerdeki herhangi bir noktanın konumunu tayin eden eğrisel parametrelerinin sinirlar1 $\left[l_{a} \in 0, b_{c}-\left(h_{f}-\rho_{1}\left(1-\sin \alpha_{n 1}\right)\right) \tan \alpha_{n 1}-\rho_{1} \cos \alpha_{n 1}\right] \quad$ ve $\left[l_{b} \in 0, b_{c}-\left(h_{f}-\right.\right.$ $\left.\left.\rho_{2}\left(1-\sin \alpha_{n 2}\right)\right) \tan \alpha_{n 2}-\rho_{2} \cos \alpha_{n 2}\right] \operatorname{dir}$.

$$
\begin{gathered}
R_{n}^{a c}=\left\{\begin{array}{l}
x_{n}^{a c} \\
y_{n}^{a c} \\
z_{n}^{a c}
\end{array}\right\}=\left\{\begin{array}{c}
\frac{\pi m_{n}}{2}-l_{a}+c_{y} \pi m_{n} \\
-h_{f}+e \\
0
\end{array}\right\} \\
R_{n}^{b d}=\left\{\begin{array}{l}
x_{n}^{b d} \\
y_{n}^{b d} \\
z_{n}^{b d}
\end{array}\right\}=\left\{\begin{array}{c}
-\frac{\pi m_{n}}{2}+l_{b}+c_{y} \pi m_{n} \\
-h_{f}+e \\
0
\end{array}\right\}
\end{gathered}
$$

Takımın $\overline{c e}$ ve $\overline{d f}$ bölgeleri imal edilen dişli çarkın kök yüzeylerini sırasıyla oluşturmaktadır. Bu bölgelerdeki herhangi bir noktanın konumunu tayin eden eğrisel parametrelerin sinirlar1 $\left[l_{c} \in 0,90^{\circ}-\alpha_{n 1}\right]$ ve $\left[l_{d} \in 0,90^{\circ}-\alpha_{n 2}\right]$ dir. $S_{n}\left(X_{n}, Y_{n}, Z_{n}\right)$ koordinat sisteminde $\overline{c e}$ ve $\overline{d f}$ bölgelerinin yer vektörleri aşağıda verilmiştir.

$$
\begin{aligned}
& R_{n}^{c e}=\left\{\begin{array}{l}
x_{n}^{c e} \\
y_{n}^{c e} \\
z_{n}^{c e}
\end{array}\right\}=\left\{\begin{array}{c}
b_{c}+\left(h_{f}-\rho_{1}\left(1-\sin \alpha_{n 1}\right)\right) \tan \alpha_{n 1}+\rho_{1} \cos \alpha_{n 1}-\rho_{1} \sin l_{c}+c_{y} \pi m_{n} \\
-h_{f}+\rho_{1}-\rho_{1} \cos l_{c}+e \\
0
\end{array}\right\} \\
& R_{n}^{c e}=\left\{\begin{array}{l}
x_{n}^{d f} \\
y_{n}^{d f} \\
z_{n}^{d f}
\end{array}\right\}=\left\{\begin{array}{c}
-b_{c}-\left(h_{f}-\rho_{2}\left(1-\sin \alpha_{n 2}\right)\right) \tan \alpha_{n 2}-\rho_{2} \cos \alpha_{n 2}+\rho_{2} \sin l_{d}+c_{y} \pi m_{n} \\
-h_{f}+\rho_{2}-\rho_{2} \cos l_{d}+e \\
0
\end{array}\right\}
\end{aligned}
$$

Takımın $\overline{e g}$ ve $\overline{f h}$ bölgeleri imal edilen dişli çarkın evolvent yanak yüzeylerini sırasıyla oluşturmaktadır. $S_{n}\left(X_{n}, Y_{n}, Z_{n}\right)$ koordinat sisteminde $\overline{e g}$ ve $\overline{f h}$ bölgelerinin yer vektörleri aşağıda verilmiştir.

$$
\begin{aligned}
& R_{n}^{e g}=\left\{\begin{array}{l}
x_{n}^{e g} \\
y_{n}^{e g} \\
z_{n}^{e g}
\end{array}\right\}=\left\{\begin{array}{c}
b_{c}-l_{e} \sin \alpha_{n 1}+c_{y} \pi m_{n} \\
l_{e} \cos \alpha_{n 1}+e \\
0
\end{array}\right\} \\
& R_{n}^{f h}=\left\{\begin{array}{c}
x_{n}^{f h} \\
y_{n}^{f h} \\
z_{n}^{f h}
\end{array}\right\}=\left\{\begin{array}{c}
-b_{c}+l_{f} \sin \alpha_{n 2}+c_{y} \pi m_{n} \\
l_{f} \cos \alpha_{n 2}+e \\
0
\end{array}\right\}
\end{aligned}
$$


$\mathrm{Bu}$ bölgelerdeki herhangi bir noktanın konumunu tayin eden eğrisel parametrelerin sinirlar1 $\left[l_{e} \in-h_{a} / \cos \alpha_{n 1}, h_{t} / \cos \alpha_{n 1}\right]$ ve $\left[l_{f} \in-h_{a} / \cos \alpha_{n 2}, h_{t} / \cos \alpha_{n 2}\right]$ dir.

\subsection{Helisel Dönüşüm}

Sn koordinat sistemine bağlı normal kesitteki profile dönme ve öteleme hareketinin bir kombinasyonu dönüşüm uygulanır. Bu dönüşüm Şekil 2'de görselleştirilmiştir ve matris ifadesi (7) numaralı denklemde verilmiştir. İmal edilen dişlinin helis açısı $\beta$ sembolüyle gösterilmektedir ve $\lambda=\left|\overline{O_{n} O_{C}}\right|$ dir.

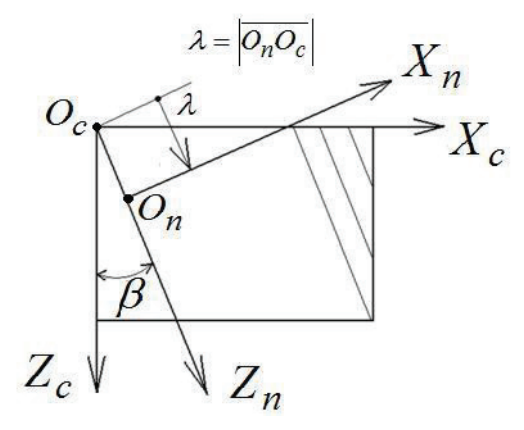

Şekil 2. Helisel Yüzey için Koordinat Dönüşüm

$$
M_{c n}=\left[\begin{array}{cccc}
\cos \beta & 0 & -\sin \beta & -\lambda \sin \beta \\
0 & 1 & 0 & 0 \\
\sin \beta & 0 & \cos \beta & \lambda \cos \beta \\
0 & 0 & 0 & 1
\end{array}\right]
$$

Böylece helisel dişli kremayer takımın vektörel ifadesi $S_{c}\left(X_{c}, Y_{c}, Z_{c}\right)$ koordinat sisteminde elde edilir.

$$
R_{c}^{i}=\left[M_{c n}\right] R_{n}^{i}
$$

$\mathrm{Bu}$ ifadede üst indis $i$ sırasıyla $\overline{a c}, \overline{b d}, \overline{c e}, \overline{d f}, \overline{e g}$ ve $\overline{f h}$ bölgelerini gösterir. Açık formda ifade aşağıda verilmiştir.

$$
\begin{aligned}
x_{c}^{i} & =x_{n}^{i} \cos \beta-\lambda \sin \beta \\
y_{c}^{i} & =y_{n}^{i} \\
z_{c}^{i} & =x_{n}^{i} \sin \beta+\lambda \cos \beta
\end{aligned}
$$


Diferansiyel geometriden takımın imal eden yüzeylerinin birim normal vektörleri aşağıdaki ifade ile elde edilir [2].

$n_{c}^{i}=\frac{\frac{\partial R_{c}^{i}}{\partial l_{j}} \times \frac{\partial R_{c}^{i}}{\partial \lambda}}{\left\|\frac{\partial R_{c}^{i}}{\partial l_{j}} \times \frac{\partial R_{c}^{i} \|}{\partial \lambda}\right\|}$

$\mathrm{Bu}$ ifadede üst indis $i$ takımın bölgelerini ve alt indis $j$ bu bölgelere ait eğrisel parametreleri gösterir.

\subsection{Dönme Düzleminde Takım}

Helisel dişli çarkın iki boyutlu modeli uygun düzenlemelerle önceki bölümlerden verilen denklemlerden elde edilir. Kremayer takımın profili dönme düzleminde ifade etmek için (9) numaralı denklemin üçüncü satırı aşağıda gösterildiği formda düzenlenir.

$\lambda=\left(z_{c}^{i}-x_{n}^{i} \sin \beta\right) / \cos \beta$

$\mathrm{Bu}$ ifade (9) numaralı denklemin birinci satırında yerine konularak $z_{c}$ nin herhangi bir değeri için dişli çarkın iki boyutlu modeli elde edilir.

\section{DISŞLI ÇARKIN MATEMATIKK MODELI}

Kremayer takım ile imal edilen dişli çark arasındaki koordinat bağı Şekil 3'de gösterilmiştir. $S_{c}\left(X_{c}, Y_{c}, Z_{c}\right)$ kremayer takımın koordinat sistemi, $S_{1}\left(X_{1}, Y_{1}, Z_{1}\right)$ imal edilen çark dişlisinin koordinat sistemi ve $S_{f}\left(X_{f}, Y_{f}, Z_{f}\right)$ sabit olan referans koordinat siste-

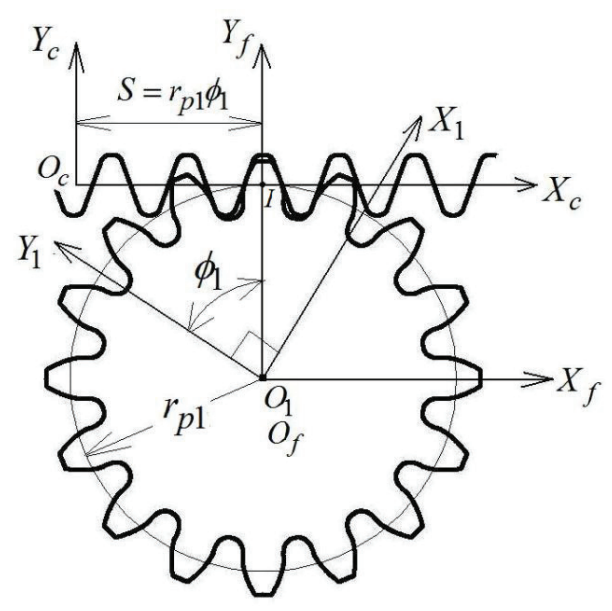

Şekil 3. Kremayer Takım ile İmal Edilen Dişli Arasındaki Koordinat Bağı 
midir. Takımın öteleme hareketi $S=r_{p 1} \varphi_{1}$ ve imal edilen dişli çarkın $\varphi_{1}$ açısı kadar dönme hareketi senkronizedir. $S_{c}$ koordinat sisteminden $S_{1}$ koordinat sistemine dönüşümün matris ifadesi (12) numaralı denklemde verilmektedir [2].

$$
\left[M_{1 c}\right]=\left[\begin{array}{cccc}
\cos \phi_{1} & \sin \phi_{1} & 0 & r_{p 1}\left(\sin \phi_{1}-\phi_{1} \cos \phi_{1}\right) \\
-\sin \phi_{1} & \cos \phi_{1} & 0 & r_{p 1}\left(\cos \phi_{1}+\phi_{1} \sin \phi_{1}\right) \\
0 & 0 & 1 & 0 \\
0 & 0 & 0 & 1
\end{array}\right]
$$

Profil kaydırılmış takımın yer vektörü $R_{1}^{i}$ ve dönüşüm matrisi olmak [ $\left.M_{1 c}\right]$ üzere (13) numaralı denklem kullanılarak takımı imal edilen dişlinin $S_{1}\left(X_{1}, Y_{1}, Z_{1}\right)$ koordinat sisteminde ifade etmek mümkündür [2].

$$
R_{1}^{i}=\left[M_{1 c}\right] R_{c}^{i}
$$

Eş çalışma denklemi Dişli Ana Kanunun matematik ifadesidir. (14) numaralı denklemde gösterilen bu ifade yuvarlanma açısı ile yüzey parametreleri arasındaki bağı tesis eder [2].

$$
\frac{X_{c}^{i}-x_{c}^{i}}{n_{c x}^{i}}=\frac{Y_{c}^{i}-y_{c}^{i}}{n_{c y}^{i}}=\frac{z_{c}^{i}-z_{c}^{i}}{n_{c z}^{i}}
$$

$X_{c}^{i}, Y_{c}^{i}$ ve $Z_{c}^{i}$ sembolleri, $S_{c}$ koordinat sisteminde, ani dönme ekseni üzerindeki bir noktanın (I noktası) koordinatlarını gösterir. Takım yüzeyindeki temas noktasının koordinatları $x_{c}^{i}, y_{c}^{i}$ ve $z_{c}^{i}$ dir. Birim yüzey normali $n_{c}^{i}$ 'nin doğrultman kosinüsleri $n_{c x}^{i}, n_{c y}^{i}$ ve $n_{c z}^{i}$ dir. $\phi_{1}$ yuvarlanma parametresi ve $r_{p 1}$ dişli çarkın taksimat dairesi yarıçapıdır.

(13) ve (14) numaralı denklemlerin eşzamanlı ele alınmasıyla imal edilen dişli çark yüzeylerinin matematik modeli elde edilir. $\mathrm{Bu}$ model ile istenilen $z_{c}$ kesitinde imal edilen dişlinin bilgisayar grafiği oluşturulabilir.

\section{EVOLVENT BÖLGE ÜST SINIRININ ANALITIK TAYINI}

Şekilde kremayer ile dişli çarkın teması alın kesitte gösterilmektedir. Temasın üst sınırı dişli çarkın baş dairesinin kavrama doğrusunu kestiği T noktasıdır. Burada $\overline{P T}$ uzaklaşma hattının uzunluğudur ve $\overline{P T}=-r_{b} \tan \alpha_{t}+\sqrt{r_{t}^{2}-r_{b}^{2}}$ olarak hesaplanır. Diş başı dairesi yarıçap $r_{t}$, temel dairesi yarıçapı $r_{b}$ ve alın kavrama açısı $\alpha_{t}$ dir. Efektif yükseklik $h_{e f f}=\overline{P T} \sin \alpha_{t}$ ifadesiyle hesaplanır. Sonuç olarak imal edilen dişli geometrisini hesaplarken takım geometrisi için verilen $\left[l_{j} \in-h_{a} / \cos \alpha_{n}, h_{t} /\right.$ $\left.\cos \alpha_{n}\right]$ yerine $\left[l_{j} \in-h_{a} / \cos \alpha_{n}, h_{e f f} / \cos \alpha_{n}\right]$ ifadesi kullanilmalıdir [10]. Profil kaydırma durumunda ise $h_{e f f}=\overline{P T} \sin \alpha_{t}-e$ olarak alınır. 


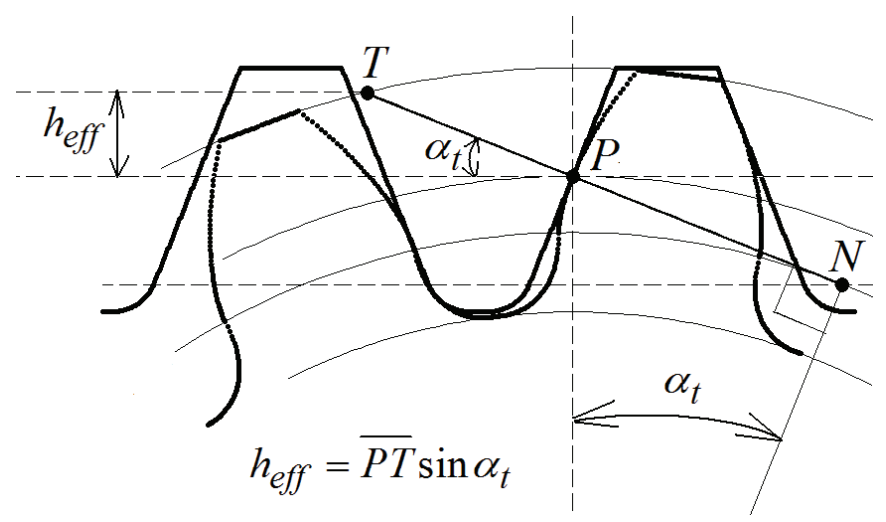

Şekil 4. Kremayer-Dişli Çark Mekanizması Kavrama Doğrusu

\section{UYGULAMALAR}

Çeşitli dizayn parametrelerinin imal edilen dişli çark geometrisine olan etkilerini incelemek için önceki bölümlerde verilen matematik modelden hareketle bir bilgisayar programı yazılmıştır. Bu hesaplayıcı program BASIC programlama dilinde GWBASIC editöründe hazırlanmış ve çalıştırılmıştır. Program çıktıları bir grafik işleme programında değerlendirilerek görselleştirilmiştir.

Şekil 5'de alttan kesilmiş bir helisel dişli çark alın kesitte gösterilmektedir. Bu uygulamada normal modül $m_{n}=3 \mathrm{~mm}$, diş sayısı $T=10$, kavrama açısı $\alpha_{n}=20^{\circ}$, helis

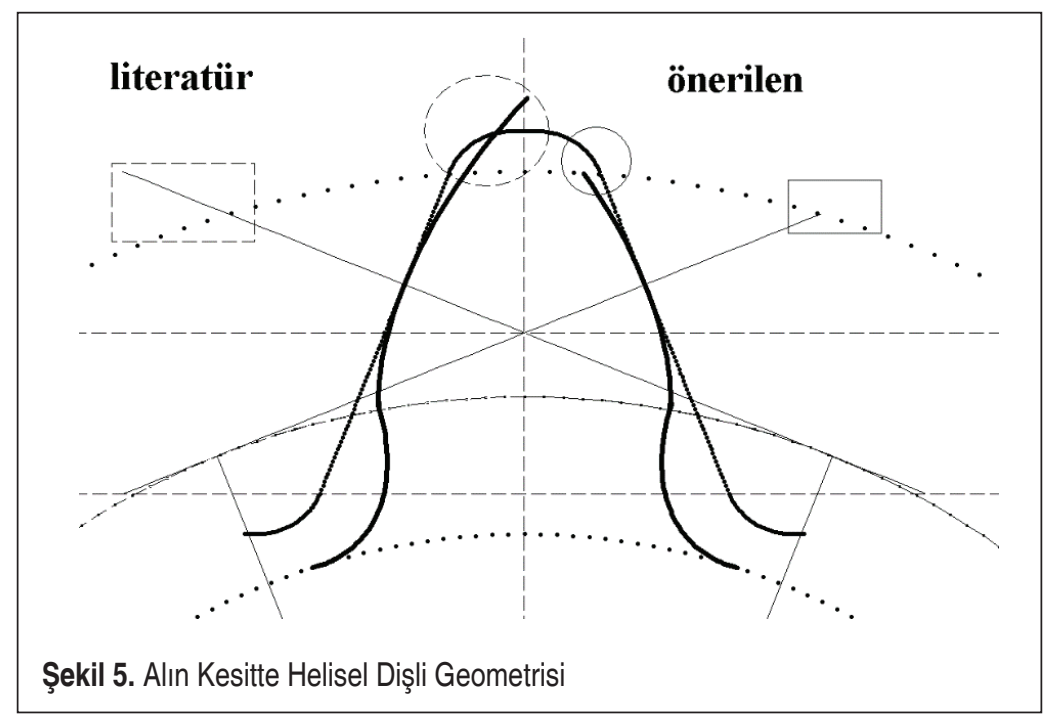


açısı $\beta=25^{\circ}$ ve takım uç yuvarlatma yarıçapı $\rho=0.38 \cdot m_{n}$ olarak alınmıştır. Burada sol profil evolvent parametrenin literatürdeki üst sınırı ile sağ profili ise önerilen üst sınır ile oluşturulmuştur. Literatürde takım için verilen üst sınır imal edilen taslakta diş başını aşmaktadır. Fazlalığı gidermek için ilave algoritma veya CAD programında trim işlemi gerekmektedir. Önerilen sınır ise analitik olarak taslak diş başı yarıçapını sağlamaktadır.

Şekil 6'da alttan kesilmiş bir helisel dişli çark alın kesitte gösterilmektedir. Bu uygulamada normal modül $m_{n}=3 \mathrm{~mm}$, diş sayısı $T=15$, kavrama açısı $\alpha_{n}=15^{\circ}$, helis açısı $\beta=15^{\circ}$ ve takım uç yuvarlatma yarıçapı $\rho=0.3373 \cdot m_{n}$ olarak alınmıştır. Şekil 7'de ise minimum profil kaydırma miktarı $e=x \cdot m_{n}=0.4452 \cdot m_{n}$ uygulanarak alttan kesmenin önlenmesi görselleştirilmiştir.
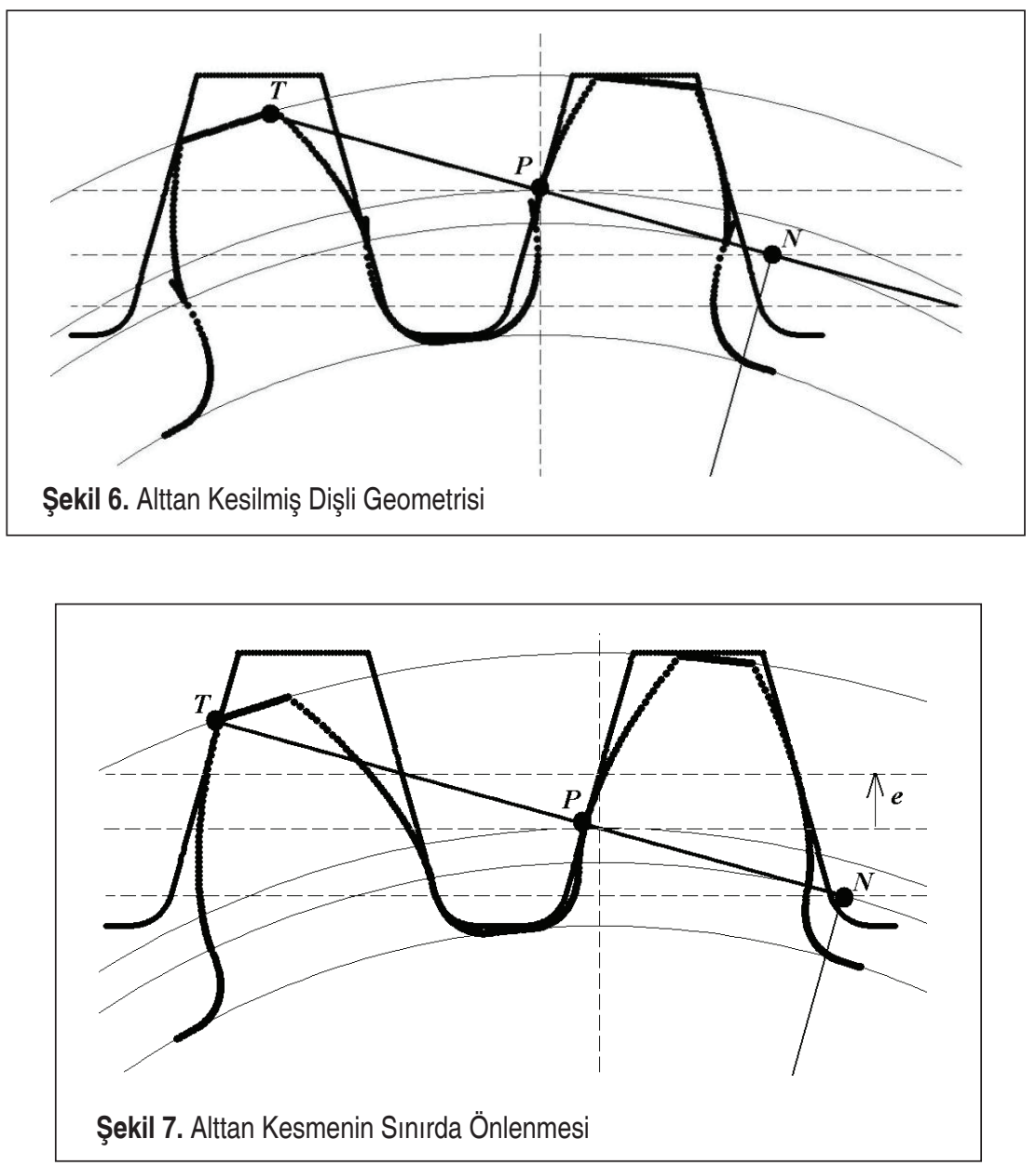
Sivri uçlu takım halinde ise sınır diş sayısı yükselmektedir. Alttan kesmenin daha belirgin olarak görüldüğü Şekil 8'deki uygulamada normal modül $m_{n}=3 \mathrm{~mm}$, diş sayısı $T=15$, kavrama açısı $\alpha_{n}=15^{\circ}$, helis açısı $\beta=15^{\circ}$ ve takım uç yuvarlatma yarıçapı $\rho$ $=0.0$ alınmıştır. Şekil 9' da ise minimum profil kaydırma miktarı $e=x \cdot m_{n}=0.6952$. $m_{n}$ uygulanarak alttan kesmenin önlenmesi görselleştirilmiştir.
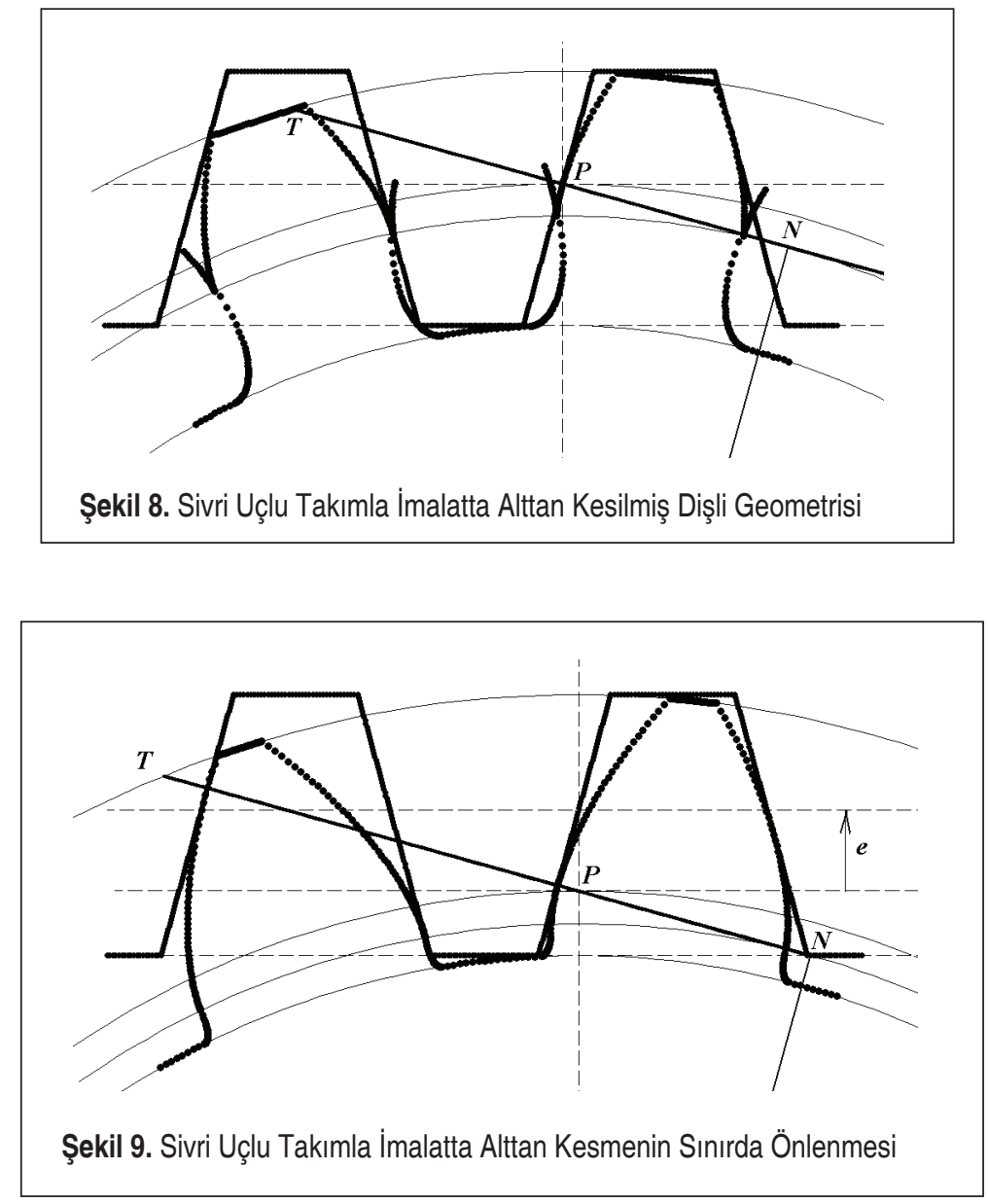

Profil kaydırmanın teorik üst sınırı sivri tepedir. Pratikte sivri tepeye müsaade edilmez. Diş başı kalınlığının belli bir değerin altında olması istenmez. Şekil 10'da $\alpha_{n}=$ $20^{\circ}$ kavrama açısı için çeşitli diş sayılarında (helisel dişliler için ise eşdeğer diş sayılarında) uygulanabilecek profil kaydırma faktörü gösterilmektedir. 


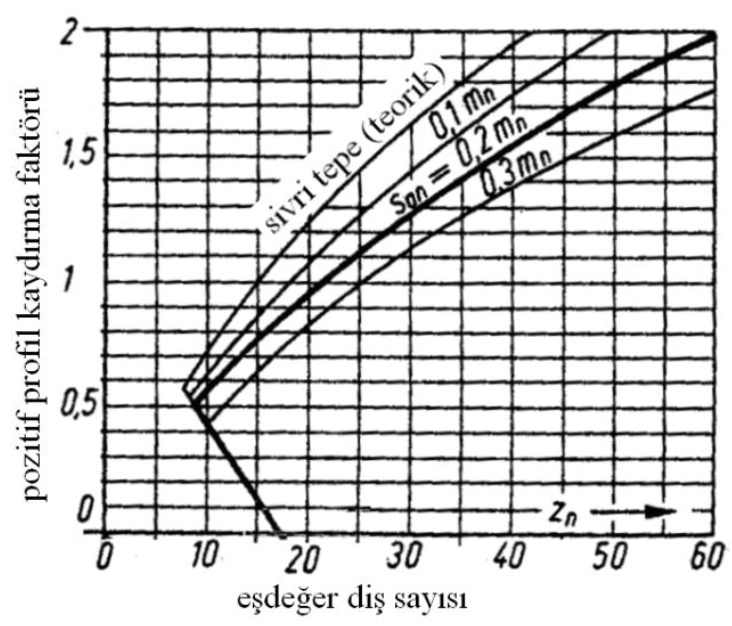

Şekil 10. Profil Kaydırma Üst Sınır Diyagramı [13]

Şekil 11'da görselleştirilen uygulamada normal modül $m_{n}=3 \mathrm{~mm}$, diş sayısı $T=12$, kavrama açısı $\alpha_{n}=20^{\circ}$, helis açısı $\beta=21.826^{\circ}$ ve takım uç yuvarlatma yarıçapı $\rho=$ $0.38 \cdot m_{n}$ olarak alınmıştır. Eşdeğer dişli çark sayısı $T_{e s ̧}=15$ dir ve profil kaydırma miktarı $e=1 \cdot m_{n}$ alınarak sivri tepe sınırına ulaşılmıştır.

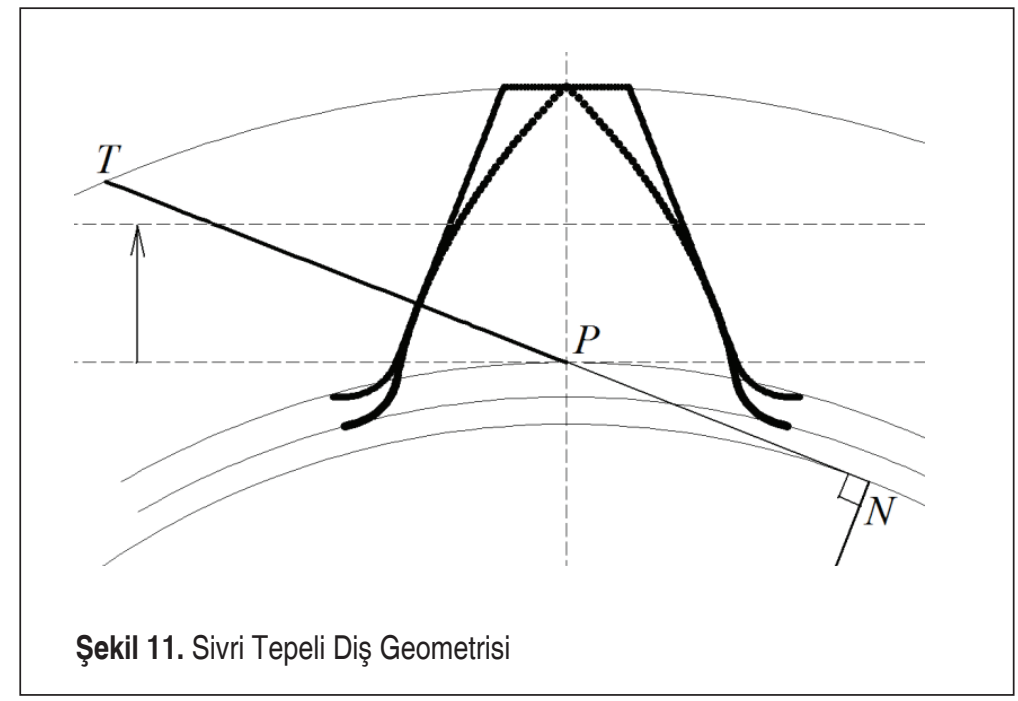


Şekil 12'de görselleştirilen asimetrik profilli dişli çark uygulamasında normal modül $m^{n}=3 \mathrm{~mm}$, diş sayısı $T=20$ ve helis açısı $\beta=15^{\circ}$ alınmıştır. Sağ profilin kavrama açıs $1 \alpha_{n 1}=20^{\circ}$ ve takım uç yuvarlatma yarıçapı $\rho_{1}=0.38 \cdot m_{n}$ dir. Sol profilin kavrama açısı $\alpha_{n 2}=15^{\circ}$ ve takım uç yuvarlatma yarıçap1 $\rho_{2}=0.3373 \cdot m_{n}$ dir. Şekil 4 'de kremayerin baş hattının (veya takımın evolvent alt sınır hattının) kavrama doğrusunu temel nokta N'de kesme durumu gösterilmişti. Şekil 12'de ise baş hattı ikinci profil için temel noktasının altında kalmaktadır. Bu durumda ikinci profilin alttan kesilmiş olduğu görülmektedir.

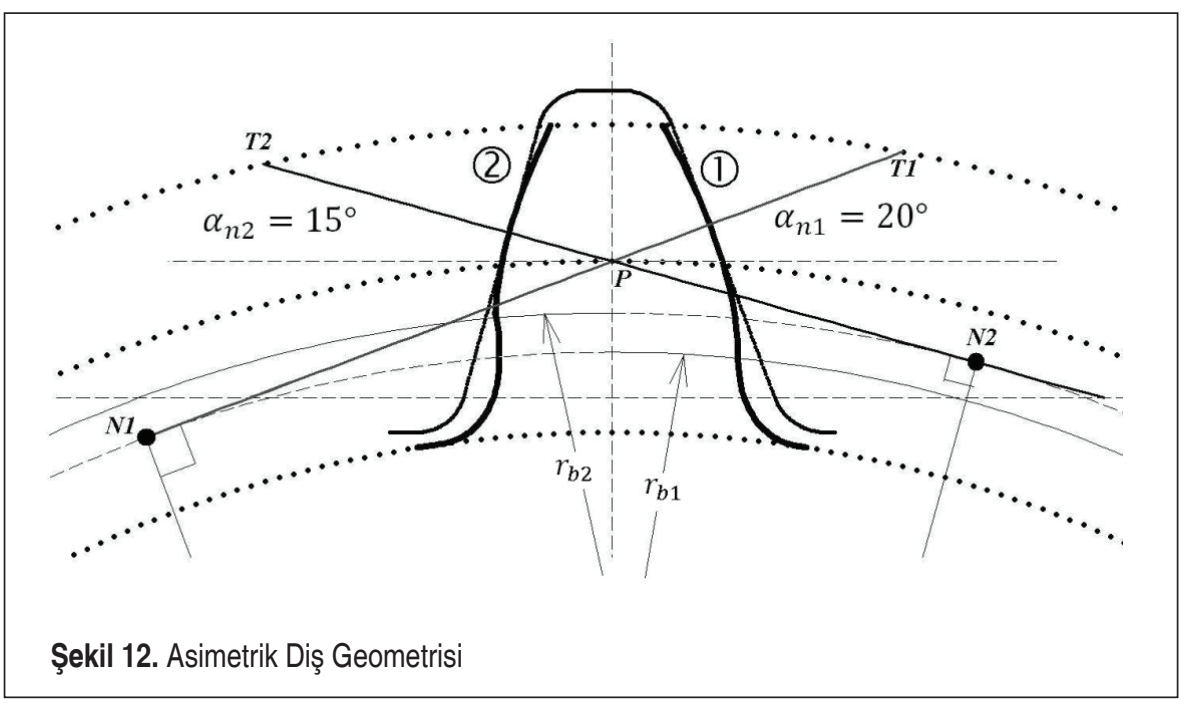

\section{SONUÇLAR}

Bu makalede evolvent profilli helisel dişli çarkların matematik modellenmesi ve bilgisayar simülasyonu ele alınmıştır. Matematik modelde asimetrik diş tasarımı da göz önüne alınmıştır. Matematik modelin programlanarak bilgisayar ortamına aktarılması ve sonuçların görselleştirilmesi tasarım aşamasında çeşitli parametrelerin imal edilecek dişlideki etkilerini inceleme firsatı sağlar. Böylelikle hatalı tasarımın yol açacağı zaman ve malzeme kaybı önlenmiş olur. Takım ucu yuvarlatma yarıçapının artması diş dibi kesitini artırmaktadır. Sivri uçlu takım ise aynı dizayn parametrelerinde alttan kesme tehlikesini artırmakta ve önlemek için daha yüksek miktarda profil kaydırmaya ihtiyaç duymaktadır. Düz dişli çark için verilen müsaade edilen profil kaydırma miktarını gösteren diyagramlar eşdeğer diş sayısı kullanılarak helisel dişli çarklar içinde geçerli olmaktadır. Profil kaydırma takımın vektör ifadesine eklenmiştir. Literatürde kremayer takımın evolvent bölgesinin üst sınırı için verilen yükseklik imal edilen dişlide diş başı sınırını aşan profil oluşturduğundan sunulan bu çalışmada analitik sınır ifadesi geliştirilmiştir. Bu ifadede profil kaydırmanın etkisi de göz önüne alınmıştır. 
Böylece standart baş dairesi dışında kalan kısımlarının kaldırılması için ilave CAD operasyonlarına veya bir algoritmaya gerek kalmamaktadır.

\section{SEMBOLLER}

$b_{c} \quad$ : Taksimat hattında takım diş kalınlığı belirleyen parametre

$\mathrm{c}_{\mathrm{y}} \quad$ : Takım diş sayısını belirleyen parametre

e : Profil kaydırma miktarı

$\mathrm{h}_{\mathrm{a}} \quad$ : Takım evolvent derinlik

$\mathrm{h}_{\mathrm{f}} \quad$ : Çark diş derinliği (takım tam baş yüksekliği)

$\mathrm{h}_{\mathrm{t}} \quad$ : Çark baş yüksekliği

$1_{i} \quad$ : Takım yüzeyi bölgelerinin eğrisel parametreleri

$\mathrm{m}_{\mathrm{n}} \quad$ : Normal modül

$\left[M_{\mathrm{ij}}\right]: \mathrm{S}_{j}$ koordinat sisteminden $\mathrm{S}_{\mathrm{i}}$ koordinat sistemine dönüşüm matrisi

$n_{c}^{i} \quad$ : Birim normal vektör

$\mathrm{r}_{\mathrm{p} 1} \quad$ : Dişli çarkın taksimat yarıçapı

S : Takımın senkron hareket miktarı

$\mathrm{S}_{\mathrm{i}} \quad$ : Koordinat sistemleri (i=f, c, 1), f sabit, c hareketli takım, 1 hareketli taslak dişli

$\mathrm{T}$ : Diş sayısı

$\mathrm{x} \quad$ : Profil kaydirma oranı

$\alpha_{\mathrm{n} 1} \quad$ : Normal kesitte kavrama açısı - sağ profil

$\alpha_{\mathrm{n} 2} \quad$ : Normal kesitte kavrama açıs1 - sol profil

$\alpha_{\mathrm{t}} \quad$ : Alın kesitte kavrama açısı

$\beta \quad$ : Helis açısı

$\phi_{\mathrm{p} 1} \quad$ : Yuvarlanma parametresi

$\rho_{1} \quad$ : Takım ucu yuvarlatma yarıçapı - sağ profil

$\rho_{2} \quad$ : Takım ucu yuvarlatma yarıçapı - sol profil

\section{KAYNAKÇA}

1. Çakır, A. 1989. Dişli Çark Kinematiği. İTÜ Makina Fakültesi.

2. Litvin, F. L. 1994. Gear Geometry and Applied Theory, ISBN: 0-13-211095-4, Prentice Hall, New Jersey, ABD.

3. Karpat, F., Dogan, O., Yuce, C., Ekwaro-Osire, S. 2017. “An Improved Numerical Method for the Mesh Stiffness Calculation of Spur Gears with Asymmetric Teeth on Dynamic Load Analysis," Advances in Mechanical Engineering, vol. 9, no. 8, doi: $10.1177 / 1687814017721856$. 
4. Tsay, C.-B. 1988. "Helical Gears with Involute Shaped Teeth: Geometry, Computer Simulation, Tooth Contact Analysis and Stress Analysis," ASME J. Mech. Design, vol. 110, no. 4, pp. 482-491.

5. Liu, C.-C., and Tsay, C.-B. 2001. "Tooth Undercutting of Beveloid Gears," ASME J. Mech. Design, vol. 123, no.4, pp. 569-576.

6. Brauer, J. 2004. “A General Finite Element Model of Involute Gears," Finite Elements in Analysis and Design, vol. 40, no. 13-14, pp. 1857-1872.

7. Chen, C.-F., and Tsay, C.-B. 2005. "Tooth Profile Design for the Manufacture of Helical Gear Sets with Small Numbers of Teeth," International Journal of Machine Tools and Manufacture, vol. 45, no. 12-13, pp. 1531-1541.

8. Yang, S.-C. 2005. "Mathematical Model of a Helical Gear with Asymmetric Involute Teeth and its Analysis", International Journal of Advanced Manufacturing Technology, vol. 26, no 5-6, pp. 448-456.

9. Huang, K. J., and Su, H. W. 2010. "Approaches to Parametric Element Constructions and Dynamic Analyses of Spur/helical Gears Including Modifications and Undercutting," Finite Elements in Analysis and Design, vol. 46, no. 12, pp. 1106-1113.

10. Fetvaci, M. C. 2017. "Determination of Effective Involute Parameter Limit in Generation Simulation of Gears Manufactured by Rack-type Cutters," Mechanics \& Industry, vol. 18, no. 4,405 .

11. Fetvaci, M. C. 2016. "Determination of Effective Involute Parameter Limit in Generation Simulation of Gears Manufactured by Pinion-type Cutters," Journal of the Faculty of Engineering and Architecture of Gazi University, vol. 31, no. 2, pp. 449-455.

12. Fetvacı, C. 2012. "Tam Dişbaşı Yükseklikli Kremayer Takımla Evolvent Düz Dişli İmalatının Bilgisayar Simülasyonu,” Mühendis ve Makina, cilt 53, sayı 635, s. 34-39.

13. Kabus, K. 2006. Maschinenelemente: Tabellen und Diagramme. ISBN: 3-446-21525-5, Carl Hanser Verlag, München. 